\title{
Self-Confidence of Nursing Students Related to their Simulation Learning Experience
}

\author{
Rasha Mahfouz $^{1,2^{*}} \quad$ Asrar Almutairi $^{1^{*}} \quad$ Elhaga Eldesouky ${ }^{3}$ \\ 1.College of Nursing, Princess Nourah bint Abdulrahman University, P.O. Box: 84428 - Postal Code: 11671, \\ Building A10, Riyadh, Saudi Arabia \\ 2.College of Nursing, AinShams University, PO Box 68, 11241 Cairo - Egypt \\ 3.School of Nursing, Port Said University, P.O. Box: 42512 Port Said, Egypt
}

\begin{abstract}
Simulated learning environments are integral to the clinical training process in many nursing programmes. Active involvement through simulation may promote critical thinking skills and increase self-confidence levels. Highfidelity and low-fidelity simulation may impact students' self-confidence differently due to the differences in their ability to project a real-life scenario. The purpose of this study was to compare the effect of high-fidelity and lowfidelity simulation on self-confidence among student nurses. The study was an analytic cross-sectional in design and took place in a public women's university in the Kingdom of Saudi Arabia, Princess Nourah University. The College of Nursing has access to both low and high fidelity simulation Center. A total of 85 participants were included in the sample and used questionnaires to compare the effect of high-fidelity and low-fidelity simulation on self-confidence among student nurses. The data analysis methods used were frequency percentages, arithmetic mean, Standard Deviation and Pearson correlation. About $74.1 \%$ of the respondents were between $19-21$ years of age, and $70.4 \%$ had a GP of 3.55-4. In addition, $95.1 \%$ had not practiced in a hospital before. Students were "hesitant" about their self-confidence after a low-fidelity simulation and "confident" after a high-fidelity simulation. The high-fidelity simulation technique had statistically significant effects on self-confidence compared to low-fidelity simulation. Nursing students who are put through the high fidelity simulation exhibit high degrees of self-confidence relative to their counterparts who are subjected to the low fidelity simulation training. Similar to the existing literature, the findings from the current research backed up this position.
\end{abstract}

Keywords: High-fidelity simulation training, Low-fidelity simulation training, Nursing Students, Patient simulation, Self-confidence.

DOI: $10.7176 / \mathrm{JEP} / 10-15-20$

Publication date:May $31^{\text {st }} 2019$

\section{Introduction}

Self-confidence, self-esteem, and self-efficacy are some of the skills that nurses need to perform effectively and adequately in clinical settings. According to Porter and colleagues, self-confidence underlies nurses' competence to perform their duties effectively. It is the responsibility of instructors in the classroom to produce nurses who are competent and confident to practice effectively in the real-world. Bandura's Social Learning Theory posits that self-confidence emanates from performing a task or behaviour, observing another person perform the task or

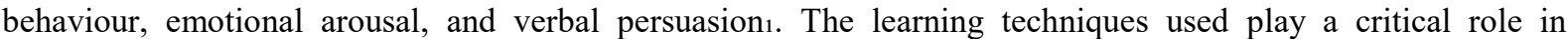
developing self-confidence among nurses. Exposure to different clinical scenarios serves to improve confidence levels among student nurses 9 . Simulation, in particular, can help students develop self-confidence.

For instance and based on a study that was conducted by Woods et al.,12, it was determined that the simulation labs played a very fundamental role in help augment the level of students' confidence. According to the study, the authors noted that nursing simulations and especially the high fidelity ones were exposing and supplying the students with the close to reality skills and knowledge before they could later transit into real life clinical settings. Nursing students are required to work on different patient scenarios under the watch of their trainers who provide these learners with real-time feedbacks and practical guidance whenever they are faced with glaring challenges as regards handling of the problem at hand. In so doing, and according to Woods et al.,12, the nursing students are able to autonomously address the "underlying problem" in a more practical and confident manner such that issues of anxiety will not arise whenever a similar scenario is presented in the real life setting.

Self-efficacy and subsequently, self-confidence is associated with good mental health. According to a study by Roddenberry and Renk $k_{10}$, self-efficacy and locus of control are some of the potential mediators of stress and illness. Karayurt and Dicle 6 also reported a positive and moderate relationship between locus of control and mental health of nursing students. The researchers reported that first and second-year students had increased risk of mental health issues due to the lower internal locus of control compared to those in advanced classes. This is primarily tied to the level of experience and exposure whereby the continued learning process is highly essential in helping a person conform and up take the different scenarios that are apparent in varying environmental settingss. In other words and within the nursing context, learning and experience over the years may serve as an advantage to senior nursing students increasing their locus of control, self-efficacy, and self-confidence compared to novice students. 
It is also important to note that practical experiences have a positive correlation with the level of self-efficacy as well as self-confidence. With increased simulation, it follows that this group of nursing students will be better prepared to transit from the environment and into the real life clinical setting as compared to those students who have not been taken through simulation, whether high fidelity or otherwise

Given the importance of self-confidence, it is important that this skill is cultivated in the classroom. Partially this is achieved by first ensuring that the nursing schools are implementing the simulation programs for their students while at the same time hiring only the qualified and skilled trainers. Based on the findings from some of the previous studies such as the one carried out by Woods et al.,12, it was determined that nurses were more confident in the clinical setting when they believed that they possessed effective skills in dealing with a case at hand. It means that nurses are likely to avoid performing a task if they think they will fail. This could be a threat to patients' lives and safety as well as the nursing profession. Consequently, it is critical to explore if the fidelity of a simulation can inspire self-confidence to help re-design teaching to cultivate self-confidence among nursing students.

Simulated learning environments are integral to the clinical training process in many nursing programmes. Simulations have been reported to enhance critical thinking skills and result in reduced anxiety and better student satisfaction. This according to Haddad and Toney-Butler ${ }_{3}$ is primarily because those nursing students exposed to simulative learning will be required to come up with real-time solutions in the simulation laboratories and other similar settings. The same will be replicated into the practical life scenarios and especially considering that at times nurses will be required to attend to patients alone in the absence of other healthcare professionals 3 . As such, critical thinking that is positively correlated with self-confidence is highly essential for the nursing professionals and as established from the preceding sections, simulative learning helps in building the two skills. It is however important to underscore the fact that, the impact on self-confidence and the differences between the effects of high and low-fidelity simulation on students' self-confidence has not been widely explored. It is important to understand if the fidelity of a simulation impacts self-confidence to enhance the classroom experience and how it translates to real life experience. Self-confidence when dealing with patients in the real world is critical and the skills to practice and master it can begin in the classroom.

Simulation educational experience is used in nursing education for psychomotor skills training. Active involvement through simulation may promote critical thinking skills and increase self-confidence levels. Highfidelity and low-fidelity simulation may impact students' self-confidence differently due to the differences in their ability to project a real-life scenario. As a result, in this study, the researchers explored the differences between low- and high-fidelity simulations on the effect of self-confidence of the students and how that prepared them to perform in real life settings. The study is significant because it can help understand the impact of the fidelity of a simulation on learners' self-confidence and assist in the designing of classrooms and learning experiences oriented to confidence in the real world. Self-confidence when dealing with actual patients in the real world is integral in the nursing care process, and these vital skills can be learned and enhanced in the classroom. Therefore, this study aiming to answer 3 specific questions:

1. How does low-fidelity simulation lab experience affect student's self-confidence?

2. How does high-fidelity simulation lab experience affect the student's self-confidence?

3. What is the difference between low-and high-fidelity simulation effect on students' self-confidence?

\section{Method}

"The study was an analytic cross-sectional design. Cross-sectional designs measure outcomes and exposures simultaneously on a group of participants selected based on inclusion and exclusion criteria 1 . This design was preferred to facilitate measuring of outcomes (self-confidence) and exposures (high-fidelity simulation versus lowfidelity simulation) at the same time. The disadvantage of this approach is that it does not derive causal

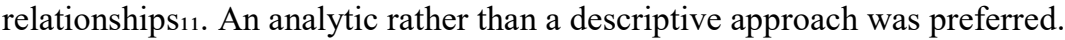

A sample was recruited from a population comprising of sixth level nursing students at a public women's university in the Kingdom of Saudi Arabia. The eligibility criteria were nursing students exposed to both highfidelity and low-fidelity simulation laboratory experiences at any single point in time and those in the sixth level. A total of 85 student participants were included in the sample. The study took place at a public women's university in the Kingdom of Saudi Arabia. The school has a College of Nursing with access to both low and high fidelity simulation techniques.

A questionnaire approach was preferred for this study. Questionnaires can collect large and diverse data from many participants simultaneouslys. Consequently, they are cost-efficient and convenient in terms of time. Due to these reasons, a questionnaire approach was preferred. The questionnaire was designed by the researcher and the reliability and validity assessed. The questionnaire's construction was based on theoretical models and readings in literature, previous studies and also considered the study problem and suggestions of the Supervisor and other experts in the field. It was agreed that the final tool should collect personal and functional characteristics of the participants in the first section where questions such as the age and competency skills practiced in a simulation 
laboratory were asked. The second section comprised of two parts, the self-confidence survey using low-fidelity simulation and self-confidence using high-fidelity simulation techniques. The first section comprised of an axis with thirteen phrases or questionsand three possible responses to the phrases, confident (with a 3-point score), hesitating ( 2 scores) and Not Confident (3 scores). The second axis had thirteen phrases meant to assess selfconfidence, the response options were also three (Confident, Hesitating and Not Confident) and the number of scores corresponded to part 1 above.

Validity describes the extent to which a tool measures what it was designed to measure. The face validity of the questionnaire used in the study was assessed using panel examination method. The Supervisor checked it for clarity, the formulation of phrases and the relevance of the phrases and suggested edits to improve the validity of the questionnaire. The internal consistency was assessed using a pilot survey. Pearson Correlation using SPSS statistical program was used to assess the internal consistency using correlation coefficients. The results were all positive and close to 1 at a $\mathrm{p}<0.01$ significance level implying that there was a high degree of internal consistency.

The reliability of a questionnaire refers to the extent to which it will yield the same results when administered to a similar population. The Cronbach's alpha was used to assess the reliability of the questionnaire utilised in the current study. The general reliability coefficient was 0.873 implying a high degree of reliability.

The data collection process began after the researcher sought Informed consent from the participants. The first questionnaire was distributed to all 85 participants during their low-fidelity laboratory practice and the second questionnaire was distributed during high-fidelity simulation lab practice. The questionnaire was in paper form and participants completed and returned the questionnaire after the session.

Ethical approval to conduct the study was obtained from the institutional review board (IRB) of the university. All students received an informed consent form with each questionnaire ensuring that the participation was optional. The questionnaire contained the purpose of the study, and a guarantee to maintain anonymity and confidentiality of the information. Participants were informed that participation was voluntary and they could withdraw from the study at any time without consequences.

\section{Result}

The data were prepared and coded for analysis. The responses, "Confident," "Hesitating" and "Not Confident" were coded as 3, 2 and 1 respectively. Arithmetic mean was used to determine the length (upper and lower limits) of the triple Scale (Confident, Hesitating and Not Confident). The highest level was 3, and the lowest level was 1. The lower level was subtracted from the higher level and divided by the number of the scale cells to obtain the correct cell of any length. For example, $(3-1=2)$ and then divided by $3(2 / 3=0.67)$. This value was added to a lower value in the scale to determine the upper limit. Based on these calculations, 1 to 1.66 represented Not Confident, 1.67 to 2.33 represented Hesitating, and 2.34 to 3 represented "Confident.". Additionally, frequency percentages were used to present the personal and functional characteristics of the study population. Weighted mean was used to determine how high or low the response options were (Confident, hesitant and Not Confident). Arithmetic mean was used to find out how high or low the responses were. Standard Deviation was used to identify the extent of deviation or dispersion of responses to study vocabulary for each phrase of the study variables phrases. Pearson correlation coefficient was used to determine the degree of correlation between the resolution and phrases axis. Only 81 out of the 85 participants returned valid questionnaires. The data were coded and analysed, and the results are presented below.

\subsection{Demographics and Descriptive Data}

\subsubsection{Age, GP and Previous Practice}

The results indicated that $74.1 \%$ of the respondents were between $19-21$ years of age, while $25.9 \%$ were aged between 22 and 24. In addition, 70.4\% of the respondents GP was 3.55-4, 22.2\% of the respondents GP was 2.503 , and $6.2 \%$ were between 4.50 and 5.0 and $1.2 \%$ between 1.50 and 2.0 . Lastly, $95.1 \%$ of the respondents had practiced in a hospital before, while $4.9 \%$ had not practiced in a hospital before. These results are presented in Table 1.

\subsubsection{Competency Skills}

About $13.6 \%$ of the respondents reported that they felt confident about their bed bathing skills after the simulation. About $11.1 \%$ felt confident in their hand hygiene, medication administration, and oxygen therapy skills after simulation. The lowest confidence levels after simulation were reported Foley Catheter skills $(2.5 \%)$ and medication calculation (3.7\%). The confident levels reported for other skills including physical assessment, initial assessment, and bed making among others are shown in Table 2.

\section{RQ1: Self-Confidence Reported in Low-Fidelity Simulation}

The mean of the self-confidence responses after low-fidelity simulation session was 1.97 of 3 . The respondents gave various responses (Confident, Hesitant and Not Confident) to the questions assessing self- confidence after a low-fidelity simulation lab session. However, the highest frequency percentage fell under "Hesitant" meaning that most respondents reported feeling hesitant after a low-fidelity simulation experience. There was homogeneity 
in the degree of approval of the respondents on the various terms in this axis. The range of the mean of the phrases of this axis was 1.88 to 2.11, which fell in the second category of the triangular scale (Hesitating). Phrase number 3 ranked first and had a mean of 2.11 out of 3 and a standard deviation of 0.689 . Phrase number 6 ranked second with a mean of 2.07 out of 3 and a standard deviation of 0.721 . Phrase number 8 ranked third with a mean of 2.06 and an SD of 0.731 . Phrase 11 ranked 13 (last) with a mean of 1.88 out of 3 and an SD of 0.765 .

\section{RQ2: Self-Confidence Reported in High-Fidelity Simulation}

The mean of the self-confidence responses after high-fidelity simulation was 2.69 out of 4 . The mean falls in the third category of the triangular scale which indicates "Confidence." This implies that the respondents reported feeling confident in various nursing skills after the high-fidelity simulation lab session. There was homogeneity in the degree of approval of the respondents on the terms used in this axis. In addition, the mean of the phrases fell between 2.63 and 2.80, which is the third category of the triangular scale indicating "Confident." Phrase number 1 (see $\backslash$ ) ranked first with a mean of 2.8 out of 3 and an SD of 0.401 . Phrase 11 ranked second with a mean of 2.74 out 0 f 3 and an SD of 0.441. Phrase 2 ranked third with a mean of 2.73 and an SD of 0.448 . Phrase 6 ranked last (13) with a mean of 2.62 and SD of 0.538.

RQ3: Difference between the effect of low-fidelity and high-fidelity simulation on Self-Confidence?

The results of the paired samples t-test used to compare effects of high-fidelity and low-fidelity simulation on selfconfidence revealed that the effect of low-fidelity and high-fidelity on self-confidence was statistically significant $(\mathrm{P}=0.01)$. The results of the t-test are presented in Table 3 . High-fidelity simulation inspired more self-confidence among the students compared to low-fidelity simulation. Assessing the association between competency skills gained in low-fidelity simulation lab versus competency skills gained during high-fidelity lab sessions revealed that significant and direct relationship. The Pearson Correlation coefficient in high-fidelity simulation as shown in Table 4 was 0.543 , meaning a positive relationship, which was also statistically significant $(\mathrm{P}<=0.001)$ while that of low-fidelity simulation was 0.288 at a $\mathrm{P}<0.009$ significance level which was not statistically significant.

\section{Discussion}

Nurses form an important segment of the society and deal with real-life issues as they are responsible of offering human services that primarily stem from human care. As such, simulation learning is highly essential in the training of the nursing professionals. Based on the already existing literature, the high fidelity and low fidelity simulations will have a different level of influence on the nursing students with regard to the way they view, conceive, and project the real-life scenarios. In the current research, the researcher sought to investigate the degree to which the high fidelity and low fidelity simulations affect the learners' self-confidence as well preparing them for the practical real-life settings. In order to achieve the objectives, there was the utilization of the cross-sectional research design and where questionnaires were used for data collection.

As expected, it was determined that the degree of fidelity had an influence on the level of self-confidence exhibited by the nursing professionals upon leaving training. For instance, and according to the findings that were obtained from the primary research, it was established that the largest number of the respondents expressed midrange confidence, whereby they were never confident nor non-confident after undergoing the low fidelity simulation training. These findings are backed up by Franklin and Lee, who concluded that the level of selfconfidence and self-efficacy was relatively low amongst those trainee nurses who had undergone through low fidelity simulation as compared to those who had been put under the high fidelity simulation.

Further and based on the findings from the current research, the researcher concluded that the nursing students who had undergone through the high fidelity lab simulation training demonstrated a wide range of nursing skills and which were highly applicable in the real-life settings. Nevertheless, this segment of the trainees exhibited high degrees of self-confidence, which again conform to the findings that were recorded by Woods et al.,12 indicating that high fidelity simulation imparted better school-industry transition skills as compared to low and medium range fidelity simulations. According to the authors, this helped enhance the level of confidence, efficacy, and creativity amongst the nurses and in which case were highly fundamental in the work environment.

As such and with reference to the degree of fidelity, the researcher concluded that self confidence amongst the nursing students was in fact a function of fidelity simulation degree. Similar to the previous studies such as the ones carried out by Haddad, Moxham, and Broadbent4; Heaveys; and Haddad and Toney-Butler, a statically significant, strong and positive correlation was established between the two variables. Apparently, the level of self-confidence, self-efficacy, as well as self-esteem plays an essential role in ensuring that the nursing professionals are able to perform their duties in the most effective manner. As such and based on the deductions made by Franklin and Lees, the nursing training institutions must put in place programs and resources that favour the positive development of the above enumerated skills amongst the nursing students. In the end, this will help alleviate the challenges faced by nurses including attrition and lack of confidence in service delivery eventually improving the overall healthcare outcomes.

It is also important to underscore the fact that the results obtained from the current research are highly applicable to research and practice. It is apparent that the researcher was able to establish valid findings that 
conform to the already existing literature, which positively links the degree of fidelity to nursing trainees' level of confidence. As such, the current research can be used as a basis of undertaking future researches as well as advising policy making amongst the stakeholders in the nursing education.

\section{Conclusion}

Owing to the central role played by the nurses coupled with an ever changing world, it is always important to focus on continued research into this area of profession. For instance, Simulation learning is technology based and technology is a highly evolving area implying that its impact on how this technique is applied is likely to change over time. It is on this basis that the current research sought to undertake an investigation into how different levels of fidelity simulation influence the degree of self confidence amongst the nursing students. The research wanted to build on different previous studies that sought to investigate the same issue. Based on the findings from most of these previous researches, it was determined that simulation learning forms a very fundamental component in the training of military experts and nursing professionals and hence the reason as to why it is increasingly being adopted in many nursing schools. A substantial number of these researches also established that the nature of simulation adopted in the training process has a direct relationship with the intended outcomes. This means that those nursing students who are put through the high fidelity simulation exhibit high degrees of self-confidence relative to their counterparts who are subjected to the low fidelity simulation training. Similar to the existing literature, the findings from the current research backed up this position.

\section{References}

Bandura, A. (1977). Self-efficacy: toward a unifying theory of behavioral change. Psychological Review, 84(2), 191. http://dx.doi.org/10.1037/0033-295X.84.2.191

Franklin, A. E \& Lee, C. S. (2018). effectiveness of simulation for improvement in self-efficacy among novice nurses: A meta-analysis. Journal of Nursing Education, 53(11), 607-614.

Haddad, L. M \& Toney-Butler, T. J. (2018). Nursing Shortage. National Centre for Biotechnology Information, $1-3$.

Haddad, M. E., Moxham, L., \& Broadbent, M. (2017). Graduate nurse practice readiness: A conceptual understanding of an old debate. Collegian, 24, 391-396.

Heavey, E. (2019). Statistics for nursing: A practical approach. Burlington, MA : Jones \& Bartlett Learning.

Karayurt, Ö., \&Dicle, A. (2008). The relationship between locus of control and mental health status among baccalaureate nursing students in Turkey. Social Behavior and Personality, 36(7), 919-930. https://doi.org/10.2224/sbp.2008.36.7.919

Masoudi, A. (2014). Self-efficacy in nursing students. Nurs Midwifery Stud, 3(4), e25881. PMC4348730

National League for Nursing (NLN). (2006). Simulation Innovation Resource Center. Retrieved from www.sirc.nln.org/mod/page/view.php?id=347

Ponto, J. (2015). Understanding and evaluating survey research. Journal of the Advanced Practitioner in Oncology, 6(2), 168. PMCID: PMC4601897

Porter, J., Morphet, J., Missen, K., \& Raymond, A. (2013). Preparation for high-acuity clinical placement: confidence levels of final-year nursing students. Advances in Medical Education and Practice, 4, 83-89. http://doi.org/10.2147/AMEP.S42157 doi: 10.2147/AMEP.S42157

Roddenberry, A., \&Renk, K. (2010). Locus of control and self-efficacy: potential mediators of stress, illness, and utilization of health services in college students. Child Psychiatry \& Human Development, 41(4), 353-370. https://doi.org/10.1007/s10578-010-0173-6

Setia, M. S. (2016). Methodology series module 3: Cross-sectional studies. Indian Journal of Dermatology, 61(3), 261. Doi: 10.4103/0019-5154.182410

Woods, C., West, C., Mills, J., Park, T., Southern, J., \& Usher, K. (2018). Undergraduate student nurses' selfreported preparedness for practice. Science Direct, 360-368. 
Tables:

Table 1. The distribution of the study sample vocabulary according to the personal and functional vocabulary

\begin{tabular}{|c|c|c|}
\hline \multicolumn{2}{|c|}{ Frequency } & Percent \\
\hline \multicolumn{3}{|c|}{ Age } \\
\hline $19-21$ & 60 & 74.1 \\
\hline $22-24$ & 21 & 25.9 \\
\hline \multicolumn{3}{|c|}{ GP } \\
\hline $1,50-2$ & 1 & 1.2 \\
\hline $2,50-3$ & 18 & 22.2 \\
\hline $3,50-4$ & 57 & 70.4 \\
\hline $4,50-5$ & 5 & 6.2 \\
\hline Yes & Have you practiced in hospital before \\
\hline No & 77 & 95.1 \\
\hline Total & 4 & 4.9 \\
\hline
\end{tabular}

Table 2. Competency Skills Reported after Simulation Experience

\begin{tabular}{|c|c|c|}
\hline Skill & Frequency & Percent \\
\hline bed making & 7 & 8.6 \\
\hline Foley catheter & 2 & $\mathbf{2 . 5}$ \\
\hline Documentation & 4 & 4.9 \\
\hline wound care & 8 & $\mathbf{9 . 9}$ \\
\hline hand hygiene & 9 & $\mathbf{1 1 . 1}$ \\
\hline Medication administration & 9 & $\mathbf{1 1 . 1}$ \\
\hline bed bathing & 11 & $\mathbf{1 3 . 6}$ \\
\hline initial assessment & 7 & 6.6 \\
\hline know signs and symptoms & 5 & 3.7 \\
\hline medication calculation & 3 & $\mathbf{1 1 . 1}$ \\
\hline Oxygen therapy & 9 & 8.6 \\
\hline physical assessment & 7 & 100.0 \\
\hline Total & 81 & \\
\hline
\end{tabular}

Table 3. Paired Samples Statistics to identify the difference between low and high fidelity simulation in the effect on self- confidence

\begin{tabular}{|l|l|l|l|l|l|l|}
\hline & N & Mean & Std. Deviation & t & df & Sig. (2-tailed) \\
\hline Low Sim.. Lab & 81 & 25.7284 & 6.97677 & \multirow{2}{*}{-7.552} & \multirow{2}{*}{80} & \multirow{2}{*}{.000} \\
\cline { 1 - 5 } High Sim. Lab & 81 & 32.4198 & 3.43098 & & \\
\hline
\end{tabular}

** Significant at the 0.01 level of significance or less

Table 4. Correlations between low-fidelity simulation and high-fidelity simulation.

\begin{tabular}{|c|c|c|}
\hline \multicolumn{3}{|l|}{ Correlations } \\
\hline & & $\begin{array}{l}\text { What were the competency skills did you } \\
\text { practice in simulation lab }\end{array}$ \\
\hline \multirow[t]{3}{*}{ Low-fidelity Sim. Lab } & Pearson Correlation & $.288^{*}$ \\
\hline & Sig. (2-tailed) & .009 \\
\hline & $\mathrm{N}$ & 81 \\
\hline \multirow[t]{3}{*}{ High -fidelity Sim. Lab } & Pearson Correlation & $.543^{* *}$ \\
\hline & Sig. (2-tailed) & 0.001 \\
\hline & $\mathrm{N}$ & 81 \\
\hline
\end{tabular}

\footnotetext{
** Significant at the 0.01 level of significance or less
} 